\title{
Inflammatory Signature in Acute-on-Chronic Liver Failure Includes Increased Expression of Neutrophil Specific Genes ELANE, MPO and CD177 Running Title: Neutrophil Transcriptomics in ACLF
}

\section{Pragyan Acharya ( $\sim$ pragyan.acharya@aiims.edu )}

All India Institute of Medical Sciences

\section{Rohini Saha}

All India Institute of Medical Sciences

\section{Shalimar}

All India Institute of Medical Sciences

\section{Prasenjit Das}

Sri Sathya Sai Institute of Higher Learning

Priyanka Mishra

All India Institute of Medical Sciences

\section{Rohan Singh}

All India Institute of Medical Sciences

\section{Sai Sanwid Pradhan}

Sri Sathya Sai Institute of Higher Learning

\section{Venketesh Sivaramakrishnan}

Sri Sathya Sai Institute of Higher Learning

\section{Research Article}

Keywords: ACLF, Neutrophils, Transcriptome, CD177, ELANE, MPO, Degranulation pathway

Posted Date: May 18th, 2021

DOI: https://doi.org/10.21203/rs.3.rs-537123/v1

License: (c) (i) This work is licensed under a Creative Commons Attribution 4.0 International License. Read Full License 


\section{Abstract}

Background and Aims: Acute-on-Chronic Liver Failure (ACLF) is associated with innate immune dysfunction and high short-term mortality. Neutrophils have been identified to influence prognosis in ACLF. Neutrophil biology is under-evaluated in ACLF. Therefore, we investigated neutrophil-specific genes and their association with ACLF outcomes.

Methods: This is an observational study. Enriched polymorphonuclear cells, containing neutrophils, isolated from study participants in three groups- $\operatorname{ACLF}(n=10)$, chronic liver disease $(C L D, n=5)$ and healthy controls $(H C, n=4)$, were analysed by microarray. Differentially expressed genes were identified and validated by qRT-PCR in an independent cohort of ACLF, CLD and HC ( $n=30,15$ and 15 respectively). The association of confirmed overexpressed genes with ACLF 28-day non-survivors was investigated. The protein expression of selected neutrophil genes was confirmed using flow cytometry and IHC.

Results: Differential gene expression analysis showed 1140 downregulated and 928 upregulated genes for ACLF vs CLD and 2086 downregulated and 1091 upregulated genes for ACLF vs HC. Significant upregulation of neutrophilic inflammatory signatures were found in ACLF compared to CLD and HC. Neutrophil-specific genes ELANE, MPO and CD177 were highly upregulated in ACLF and their expression was higher in ACLF 28-day non-survivors. Elevated expression of CD177 protein on neutrophil surface in ACLF was confirmed by flow cytometry. IHC analysis in archival post mortem liver biopsies showed the presence of $\mathrm{CD} 177^{+}$neutrophils in the liver tissue of ACLF patients.

Conclusions: Neutrophil-specific genes ELANE, MPO and CD177 are highly overexpressed in ACLF neutrophils as compared to CLD or HC. Further, these genes are highly overexpressed in ACLF 28-day nonsurvivors.

\section{Introduction}

Acute-on-chronic liver failure (ACLF) is known to be driven by systemic inflammation, and is associated with multiple organ dysfunction leading to a very high short-term mortality ${ }^{1}$. Mortality for ACLF within a 28-day period is almost $50 \%{ }^{1}$. Neutrophils form a major component of the innate immune system and the neutrophil-to-lymphocyte ratio (NLR) has been shown to be a predictor of mortality in ACLF ${ }^{2-6}$. Recent studies reveal certain characteristics of neutrophil phenotypes in ACLF such as reduced, phagocytosis, increased expression of CXCR1/2, differentially expressed cell surface TLR, enhanced NET production ${ }^{7-}$ 10. However, in-depth molecular understanding of neutrophils and their relevance in ACLF outcomes is lacking. Therefore, the objective of the present study was to define the transcriptomic profiles of polymorphonuclear cells derived from ACLF patients, to identify neutrophil specific gene signatures from the transcriptome and, to explore association between expression of neutrophil genes with ACLF 28-day mortality. 
We provide a detailed analysis of differentially expressed genes in ACLF derived polymorphonuclear cells (PMN), that contain neutrophil specific genes, vs those of chronic liver disease (CLD) patients and healthy controls $(\mathrm{HC})$. Using a combination of experimental and analytical approaches, we identify ELANE, MPO and CD177 as consistently upregulated genes in the PMN of ACLF vs CLD and HC. We also found that this neutrophil-specific gene signature was higher in ACLF 28-day non-survivors as compared to survivors. We have also analyzed the pathways that characterize the ACLF PMN as compared to CLD and $\mathrm{HC}$.

\section{Materials And Methods}

\section{Study Groups and sample collection}

ACLF patients admitted to the Department of Gastroenterology, All India Institute of Medical Sciences New Delhi, were diagnosed as per the APASL (Asian Pacific Association for the Study of the Liver) criteria and recruited into this study ${ }^{1}$. The patient recruitment period was between April 2018- November 2019. The grades of ACLF were defined according to the EASL definition and categorized as grade 1,2, and 3 depending on the number of organ systems involved. ACLF 1: patients with renal failure (creatinine $\geq 2.0 \mathrm{mg} / \mathrm{dl}$ ) or a non-renal organ failure plus renal dysfunction (creatinine between $1.5-1.9 \mathrm{mg} / \mathrm{dl}$ ) and/or HE grade I-II. ACLF 2: Patients with 2 organ failures; ACLF 3: Patients with 3 or more organ failures ${ }^{11}$. Patients with hepatocellular carcinoma or portal vein thrombosis, age group of $<18$ years and $>75$ years, diabetes (defined on the basis of recent (within 3 months) available fasting blood sugar ${ }^{3} 126$ $\mathrm{mg} / \mathrm{dl}$ or random blood sugar ${ }^{3} 200 \mathrm{mg} / \mathrm{dl}$; were excluded from the study. During the current admission a random blood sugar $\geq 200 \mathrm{mg} / \mathrm{dl}$ ( $11.1 \mathrm{mmol} / \mathrm{l})$ was used to define diabetes) ; presence of prior renal, respiratory and/or cardiovascular disease were excluded. Patients with impaired blood glucose levels were excluded because hyperglycaemia even without diabetes has been shown to alter basal neutrophil

metabolism and cause activation ${ }^{12-14}$. ACLF patient samples were collected on the day of admission, and follow-up of 28-days was done. After 48 hours of admission, based on the blood culture reports, ACLF patients were stratified as those with sepsis or without sepsis (sterile inflammation). After the 28-day follow up period, ACLF patients were stratified as survivors and non-survivors.

Chronic liver disease (CLD) patients who were within the age group $>18$ years and $<75$ years, treatment naïve, ambulatory, without any extra-hepatic complications, and no overt symptoms were recruited from the outpatient department. Acute decompensation of CLD but without ACLF (CLD-AD) was defined as complications of cirrhosis such as jaundice, ascites, hepatic encephalopathy and GI bleed, without associated organ failure ${ }^{15}$.

Age and gender-matched healthy volunteers with no recent infection or history of past chronic illness were recruited as study controls. The study has been approved by the institute ethics committee [Reference No. IEC/473/9/2016 and, IEC/369/7/2016].

\section{Sample processing}


PMN were isolated from $6 \mathrm{ml}$ of whole blood in EDTA within 2 hours of collection, followed by centrifugation at $500 \mathrm{Xg}$ for 10 minutes. Plasma was aseptically separated and stored at $-80^{\circ} \mathrm{C}$ until further use. The remaining blood pellet used for PMN isolation as described below. Enriched PMN were used for all experiments. Overall workflow is described in Figure 1A.

\section{PMN Isolation and Enrichment Analysis by Flow Cytometry}

PMN were isolated by modified Boyum's method of double gradient centrifugation ${ }^{16}$. Blood pellet containing the buffy coat was diluted with 2X volume of sterile 1X PBS (VWR, USA, 97062-730), at room temperature (RT). Ficoll-Hisep (Himedia, INDIA, LSM 1077) was layered over Granulosep (Himedia, INDIA, LSOO4) in a 2:3 ratio to prepare a double gradient was prepared in a $15 \mathrm{ml}$ centrifuge tube. Whole blood was carefully layered on top followed by centrifugation at $300 \mathrm{Xg}$ for 30 minutes at RT without brakes. The following phases were formed in order (top to bottom) after centrifugation: Diluted plasma, PBMC, Hisep, PMN, Granulosep, RBC pellet. Diluted plasma was discarded; PBMC layer was separated and the lower enriched PMN layer was collected. PMN cells were resuspended in sterile 1X PBS and washed twice by centrifugation at $500 \mathrm{Xg}$ for 10 minutes. Contaminating RBCs were removed by incubating the washed pellet in $1 \mathrm{X} \mathrm{RBC}$ lysis solution for 10 minutes at $4^{\circ} \mathrm{C}$, followed by two $1 \mathrm{X}$ PBS washes. The final cell pellet was reconstituted in $1.2 \mathrm{ml}$ filtered RPMI-1640 cell culture media (Himedia, INDIA, AL 171A) supplemented with $2 \%$ heat-inactivated fetal bovine serum (FBS) (Himedia, INDIA, RM10432). The enriched PMN cells were diluted 1:20 and counted by Trypan blue exclusion assay to score for live and dead cells $(1 \mu \mathrm{l}$ of reconstituted cells with $10 \mu \mathrm{l}$ of $0.4 \%$ of Trypan blue and $9 \mu \mathrm{l}$ of sterile $1 \mathrm{X}$ PBS). Total cells were counted, and $1 \times 10^{5}$ cells were stained with respective antibodies. To assess PMN enrichment, anti-human CD14 (clone M5E2, FITC conjugated, Cat No.301803, Biolegend, USA) and anti-human CD16 (clone 3G8, APC conjugated, Cat No 302011, Biolegend, USA) were used and their respective stain index calculated by antibody dilutions. $5 \times 10^{4}$ cells were acquired in BD LSR Fortessa X-20 Flow Cytometer. Unstained controls and single-stain tubes were prepared for each stained sample, and the acquisition was supported by BD FACSDiva software. PMN cells were gated using SSC vs FSC plot and single cells were gated as FSC height vs. FSC area (Figure $1 \mathrm{~B}-\mathrm{E}$ ). Neutrophils were selected as CD14- (negative) CD16 ${ }^{+}$ (positive) population. The percentage enrichment of samples is listed in the Supplementary Table 1.

\section{PMN RNA isolation and microarray}

RNA isolated from $1 \times 10^{6}$ enriched PMN cells was subjected to microarray to identify differentially expressed genes. Briefly, RNA was isolated from $1 \times 10^{6}$ cells using the TRIzol (Invitrogen) and the manufacturer's protocol was followed. The cell suspension was pelletized and $1 \mathrm{ml}$ TRIzol was added to solubilize the cells by vortexing for 30 seconds. Molecular biology grade chloroform $(0.2 \mathrm{ml})$ was added and mixed until milky-white appearance was formed and incubated at room temperature for 10 minutes. Phase separation was done by centrifugation at $12,700 \mathrm{Xg}$ for 15 minutes. The upper aqueous phase was removed carefully, and RNA was precipitated using $400 \mu \mathrm{l}$ of ice-cold Isopropanol. The precipitate was washed in $75 \%$ ethanol before being air-dried and suspended in $30 \mu \mathrm{l}$ of RNase free water. RNA quantity and quality were checked using Bioanalyzer. 
Total RNA with satisfactory integrity (RNA integrity number or RIN>7.0) and concentration, were labelled and cRNA was synthesized using Agilent Low Input Quick Amp Labelling Kit, One-Colour, without spike-in. This was followed by purification by RNeasy Mini Kit which was quantified with Nanodrop spectrophotometer (Thermofischer Scientific). The RNA yield and specific activity of Cy3 were calculated and samples meeting the manufacturer's threshold were prepared for hybridization. $240 \mathrm{ng}$ of labelled cRNA was hybridized using the Agilent Gene expression hybridization kit, onto SurePrint G3 Human gene expression v3 (Cat no. G4851C) chips, at $65^{\circ} \mathrm{C}$ for 17 hours as per the manufacter's protocol. The chips were washed and scanned using the Agilent surescan microarray scanner.

\section{Gene expression Data analysis}

Microarray data have been recorded and prepared according to Minimum Information About a Microarray Experiment (MIAME) guidelines and raw data have been submitted at NCBI Gene Expression Omnibus (GEO) with the accession number GSE156382. Microarray data were extracted using the Agilent Feature extraction software. The 23 individual sample feature extracted files (FEF) were converted, and data normalization was done using Genespring software followed by data analysis (Version 7.0). Briefly, the FEF files containing 56,000 probes individually, was exported to Genespring software, and sample order was chosen and grouping was done (ACLF, CLD, Healthy, ACLF sepsis, ACLF sterile inflammation). Data normalization was done using Percentile and background correction was done based on median. The normalized data was subject to sequential filtering: Expression filter of 20-100 window, Filter on flags of Detected/Non detected, Filter on Error on $\mathrm{CV}<50 \%, \mathrm{SD}<0.1, \mathrm{SD}<0.5$. Statistical analysis was proceeded with the probe-set of $\mathrm{CV}<50 \%$. NetworkAnalyst (https://www.networkanalyst.ca/NetworkAnalyst/home.xhtml) was used for carrying out DEG analysis of the mentioned sample comparatives. NetworkAnalyst uses Limma workflow for analysing data from gene expression experiments ${ }^{17}$. The differentially expressed genes were then selected with threshold criteria of $p$-value $<0.05$ and log FC $>1.0$ and $<-0.5$. The significant DEG were then tested for their biological pathway implication. All the gene sets from respective comparisons were taken individually for enrichment analysis. The enrichment analysis was done using ClueGO (a Cytoscape plugin) and Enrichr 18-20. The enrichment criteria for ClueGO was set for detailed network specificity at $5 \%$ genes for clustering and pathway significance of $p$-value $<0.05$. For Enrichr, the significant pathways were selected at adjusted p-value $<0.05$. KEGG enrichment analysis was used for overlapping of the DEG in both cases. Network visualization for enriched pathways. The pathways output from ClueGO was used for visualization in Cytoscape. A network centrality analysis was done for the created pathway with CytoNCA. A custom style was created wherein genes involved in various pathways were highlighted based on their expression levels and, the size of individual nodes varying to their betweenness values, which helped identify the key elements (genes/pathways) that regulate the network created. Gene set enrichment analysis (GSEA) was additionally performed using the Molecular Signatures Database or MSigDB (http://www.gsea-msigdb.org/gsea/msigdb/index.jsp).

\section{Microarray validation and tissue-level expression by Quantitative Real-time PCR}


Total PMN RNA was reverse transcribed into cDNA using verso cDNA synthesis kit (AB-1453A from Thermo Fisher Scientific). DNasel treatment was inherent to this kit and prevented genomic DNA carryover into downstream reactions. PCR and qRT-PCR were performed with $500 \mathrm{ng}$ of CDNA synthesized. from total PMN RNA. Prior to qRT-PCR, temperature gradient standardization for all primer sets were done to select optimum annealing temperature. Validation of gene expression log fold change by qRT-PCR was performed for the top 8 differentially expressed genes in ACLF vs CLD using specific primers designed from IDT oligoanalyzer software. The upregulated genes chosen for validation were ELANE, MPO, CD177, OLFM4, and OLAH. The transcript for 18S rRNA was used as a reference gene. The genes ELANE, MPO and CD177 were selected on the basis of their reported specificity in neutrophils, since the neutrophil population is known to be expanded in ACLF, resulting in a high NLR ${ }^{21-24}$.

The primer sequence and qRT-PCR conditions are included as supplementary data as per Minimum Information for Publication of Quantitative Real-Time PCR Experiments (MIQE) guidelines (See Supplementary data_MIQE guidelines file). All graphical representations of log fold change gene expression were done using Graphpad Prism.

\section{Cell-surface CD177 Staining and Detection by Flow Cytometry}

Peripheral blood was collected in EDTA vial, and $200 \mu \mathrm{l}$ of blood was aliquoted for antibody staining. Briefly, $1 \mathrm{ml}$ of RBC lysis buffer was added to $200 \mu$ of whole blood, and mixed properly. A $10 \mathrm{X}$ red blood cell lysis buffer was prepared in-house using $\mathrm{NH}_{4} \mathrm{Cl}(0.155 \mathrm{M}), \mathrm{KHCO}_{3}(0.01 \mathrm{M})$ and EDTA $(0.1 \mathrm{mM})$. The tubes were incubated for 10 minutes at $4^{\circ} \mathrm{C}$, and centrifuged at $300 \mathrm{xg}$ for 10 minutes. The cell pellets were washed twice with 1X PBS and suspended in $300 \mathrm{ul}$ of PBS to obtain a single cell suspension. For cell surface staining, $100 \mu \mathrm{l}$ of cell suspension was used, and the antibodies CD16 (1:100) (clone 3G8, APC conjugated, Cat No 302011, Biolegend, USA), CD66b (1:100) (clone G10F5, FITC conjugated, Cat No 305103, Biolegend, USA) and CD177 (1.5:100) (clone MEM-166, APC/Cyanine 7 conjugated, Cat No 315809, Biolegend, USA) was used. Unstained controls, stained samples, and fluorescence minus one controls were acquired on BD-LSR Fortessa flow cytometry machine. Using the FACS DIVA software, PMN gating was done based on FSC-A v/s SSC-A plot. Enriched and activated neutrophils were gated based on $\mathrm{CD} 16^{+} \mathrm{CD} 66 \mathrm{~b}^{+}$(double positive) in a quadrant plot. $\mathrm{CD} 177^{+}$neutrophils were gated within these double positive cells.

\section{Dual colour Immunohistochemistry for CD177 and CD16 in Post-Mortem Liver Biopsy}

5-micron thick sections of formalin-fixed paraffin-embedded (FFPE) tissues were retrieved from the Department of Pathology, AlIMS New Delhi and taken on coated slides. Deparaffinization was done by dipping the slides in xylene for 5 mins (2 changes), acetone for 2-3 mins, alcohol for 2-3 mins, and then under running/tap water. Antigen retrieval was performed with citrate buffer $(\mathrm{pH}=6)$ in a microwave oven, at 100 degrees Celsius at $900 \mathrm{MW}$ for 30 mins. Tissue sections were then allowed to cool down to come to room temperature. The slides were washed three times with Tris buffer $(\mathrm{pH} 7.5)$. Endogenous peroxidase blocking was done with $4 \%$ Hydrogen peroxide in $96 \mathrm{ml}$ of methanol for $20 \mathrm{~min}$. Anti-CD177 
(Invitrogen, pH 9, 1: 50), Rabbit anti-human antibody was incubated overnight at 2-4 degrees Celsius. Next day, three sequences of washings were given with Tris buffer $(\mathrm{pH} 7.5)$. Universal polymer-based secondary antibody (SkyTek Laboratories, USA) was incubated at room temperature for 30 minutes, and the reaction product was developed with 3, 3"-diaminobenzidine chromogen (1: 1). Appropriate positive and negative controls were used. Colour development was monitored under the microscope. Four subsequent sequences of washings with TRIS buffer were given at 5 minutes intervals, followed by the addition of 200 microliters of enhancer and incubation for 5 minutes at room temp. After that the Mouse anti-CD16 antibody (Invitrogen, USA. 1: 50) was incubated at room temperature for $1 \mathrm{hr}$. Three sequences of washings were given with TRIS buffer. Alkaline phosphatase tagged goat anti-mouse IgG H\&L secondary antibody (Ab7069) was added in a dilution of 1:10 for 30 mins at room temperature. Three sequences of washings were given in Tris buffer. A VECTOR® Blue Alkaline Phosphatase chromogen was used to develop the colour of the reaction (Blue AP), prepared in Tris HCL, pH 8.5, with 5 minutes incubation at room temperature and monitoring under the microscope. The slides were then washed under running tap water for 3-5 mins, counterstained with Neutral red before mounting with a glycerin solution. The dual-colour stained slides were photographed by using a BX43 Olympus microscope. The images taken at $2 x$ objective power were divided into multiple fields of vision (FOVs) having a diameter of $2 \mathrm{~mm}$ each. The procedure for manual cell counting the biopsy core was sequentially divided into multiple non-overlapping FOVs. A systematic eyeballing and counting of the cells were performed and noted. This was done to adjust the variable number of FOV available for each case due to varying biopsy core lengths. In the case of clustering and overlap of the stained cells, only those cells were counted whose nuclei were identified. Additionally, in each case, randomly in 5 FOVs the manual counting was crosschecked by the manual tagging and counting tool of 'the Image Proplus 6.1 software.

\section{Statistical Analysis}

All statistical analyses and graphical representations were done using Graphpad Prism software. Normally distributed continuous variables were expressed as Mean \pm SD, and continuous variables with skewed distribution were expressed as Median (Interquartile Range). For two group comparisons, Unpaired T-test and Mann-Whitney Test were applied for categorical variables and non-normally distributed variables, respectively.

\section{Results}

\section{Study Participants, Workflow and Neutrophil Enrichment}

The study groups included in the microarray-based gene expression analysis were ACLF ( $n=10)$, CLD $(n=6)$; as well as Healthy controls $(n=4)($ Table 1, Figure $1 A)$. ACLF is a dysfunction that occurs due to an acute injury over an underlying chronic liver disease (CLD). All the ACLF patients recruited into this study had organ failure scores of above 2 (CLIF-C- OF 2 and 3) indicating the presence of multiple organ dysfunction. Chronic etiologies in ACLF patients included alcohol $(n=4)$, autoimmune hepatitis $(A I H ; n=3)$, cryptogenic causes $(n=3)$. Acute etiologies included alcohol $(n=2)$, sepsis $(n=3), A l H(n=1), A l H+\operatorname{Sepsis}$ 
$(n=1), H E V+$ alcohol $(n=1)$, unknown causes $(n=2)$. Etiologies for CLD (Compensated -diseased controls) patients included alcohol $(n=3)$ and viral hepatitis $(n=3)$ (Table 1$)$.

Neutrophil enrichment was determined by surface labelling of cells for the markers CD14 and CD16; followed by flow cytometric analysis (Figure 1B-E; Supplementary Table 1). Neutrophil enrichment was calculated as the percentage of $\mathrm{CD} 16^{+}$cells in the PMN gate over total acquired cells. Purity of the neutrophil population was defined as the percentage of $\mathrm{CD} 16^{+}$cells over total PMN. The median values for PMN enrichment were: ACLF 80.60 \% (45.1-93); CLD 52.3 \% (41.2-60); HC 75.7\% (68.5-82.7). The median values for $\mathrm{CD} 16^{+}$neutrophils as a percentage of total PMN were - ACLF 97.9\% (67-99) ; CLD 84\% (76-95) ; HC 96 \% (90-98-98.79) (Supplementary Table 1). RNA isolated from enriched neutrophils were subjected to microarray analysis. Differential gene expression (DGE) and pathways analysis were performed only with the samples which showed $>50 \%$ enrichment and RIN score $>6.0$ (Figure 2A-D). The only exceptions were- 1 sample of ACLF (45.2\%) and 2 of CLD (45.2 \% and $41.1 \%)$. These were included due to their high RIN value (>7.0) suggesting good RNA quality. Since the aim of the experiment was to describe an overall gene expression profile of PMN cells and additional validation of selected neutrophilspecific genes using qRT-PCR was incorporated, these three samples were included so that robust transcriptome profiles could be obtained from which gene expression patterns could be further extracted and validated.

\section{Differential Gene Expression Patterns in ACLF, CLD and HC}

Differential gene expression (DGE) analysis revealed 2068 (1140 downregulated and 928 upregulated) genes for ACLF vs CLD and 3177 (2086 downregulated and 1091 upregulated) genes for ACLF vs HC (Figure $2 \mathrm{~A}-\mathrm{C}$ ). CD177 was one of the highest upregulated genes in ACLF vs HC as well as ACLF vs CLD (Figure $2 \mathrm{C}$ ). Other genes found to be among the top 50 upregulated genes in ACLF were- ELANE, OLFM4 and OLAH (Figure 2C; Supplementary Tables 2 and 3). MPO gene, a classical neutrophil activation marker was found to be upregulated in ACLF vs CLD with a Log2FC of 2.4 (Supplementary Table 2). Based on the neutrophil gene expression patterns reported in literature, and log-fold values in our microarray data set, 5 differentially expressed genes from ACLF vs. CLD, were selected for validation using qRT-PCR which included ELANE, MPO, CD177, OLFM5 and OLAH (Figure 2D). Overall trends for ACLF vs. CLD were found to be conserved in the qRT-PCR analysis (Figure 2D). ELANE, MPO and CD177 are three genes which have been linked to neutrophil pathogenicity in inflammatory disorders and were found to be significantly upregulated in ACLF PMN compared to CLD or HC (Figure 2E).

\section{Association of PMN Gene Expression with Short-Term Mortality in ACLF}

Since the high degree of mortality observed in ACLF is believed to be driven by highly inflammatory innate immune responses, gene expression levels obtained from qRT-PCR experiments for the genes ELANE, MPO, CD177, OLFM4, OLAH (upregulated in ACLF vs CLD or ACLF vs HC) were stratified based on 28-day mortality in ACLF (Figure $3 \mathrm{~A}-\mathrm{E}$ ). Mean gene expression values of the neutrophil-specific genes ELANE, MPO and CD177 were significantly elevated in 28-day non-survivors as compared to survivors 
(Figure 3 A-C; $p$ values $0.004,0.01,0.03$ respectively for ELANE, MPO and CD177). OLFM4 and OLAH were comparable in both the groups amongst survivors and non-survivors with ACLF (Figure $3 \mathrm{D}, \mathrm{E}$ ). Stratification based on sex showed comparable values of ELANE, MPO, CD177, OLFM4 and, OLAH in both males and females (Supplementary Figure 1) in all the three groups (ACLF, CLD and HC).

Since sepsis is a major etiology as well as complication in ACLF, gene expression values were stratified into sepsis vs sterile inflammation (Supplementary Figure 2). ELANE, MPO, CD177, OLFM4 and OLAH gene expression were found to be comparable in both the groups sepsis and sterile inflammation (Supplementary Figure 2) indicating that neutrophil response to both bacterial infection and tissue injury involved upregulation of these genes.

\section{CD177 surface level expression is elevated in ACLF}

CD177 is a neutrophil specific cell surface molecule which defines heterogeneous neutrophil populations. Since the CD177 gene was found to be highly overexpressed in ACLF neutrophils, we investigated the cellsurface protein level expression of CD177 in circulating neutrophils from ACLF patients. For this, the total percentage of $\mathrm{CD} 16^{+}$and $\mathrm{CD} 66 \mathrm{~b}^{+}$neutrophils were estimated from the whole blood of ACLF, CLD and Healthy ( $n=10$ per group) samples. These enriched double positive $\left(C D 16^{+} C D 66 b^{+}\right)$cells define mature and activated neutrophils in circulating blood. ACLF, CLD and HC groups included in the flow cytometric analysis of CD177, had median enrichment values of $81.6 \%$ (62.7-94.3), 97.4\% (95.3-98.5) and 90.3\% (84.1-94.1) \% respectively (Figure $4 \mathrm{~A}$ ).

$\mathrm{CD} 177^{+}$cells were gated within the $\mathrm{CD} 16^{+} \mathrm{CD} 66 \mathrm{~b}^{+}$positive neutrophil population (lineage markers for granulocytes and neutrophils) for all samples. Percentage of $\mathrm{CD} 177^{+}$neutrophils as a proportion of total $\mathrm{CD} 16^{+} \mathrm{CD} 66 \mathrm{~b}^{+}$neutrophils were compared between the study groups. Percentage of $\mathrm{CD} 177^{+}$neutrophils in total $C D 16^{+} \mathrm{CD} 66 \mathrm{~b}^{+}$neutrophils were found to be significantly higher in ACLF ( 80\%) as compared to CLD $(\sim 50 \%)$ (p-value $<0.0001)$ and Healthy controls $(\sim 60 \%)$ (p-value 0.005$)$ (Figure 4 B, C). The values of $\mathrm{CD} 16^{+} \mathrm{CD} 66 \mathrm{~b}^{+}$enrichment and CD177 positivity for individual samples are provided in Supplementary Table 4.

\section{Presence of CD177+ Neutrophils in ACLF and CLD-AD Post-Mortem Liver Biopsies}

Formalin fixed paraffin embedded (FFPE) liver biopsies from deceased ACLF and CLD-AD (CLD with acute decompensation but without ACLF) were retrospectively retrieved and subjected to dual colour IHC for CD16 and CD177 in order to investigate the tissue localization of the CD177 ${ }^{+}$neutrophil subpopulation (Figures 5 A-D). A limitation of this experiment was that "normal" or "CLD" control liver biopsies are ethically non-permissible at our centre in the absence of clinical indication for a biopsy in live patients, and therefore, cannot be included as controls. The CD177 stained neutrophils were represented by brown stain (black arrows) and the CD16 stained neutrophils, monocytes, macrophages, and Kupffer cells were represented by blue stain (blue arrows) (Figures $5 \mathrm{~A}, \mathrm{~B}$ ). The manual counts, post validation was averaged for each cell type in a case and were expressed per $3.14 \mathrm{~mm}^{2}$, that is the area of each FOV 
of 10x objective in this microscope (Supplementary Table 5). These data show that CD $177^{+}$neutrophils are detectable in the liver tissue of the deceased patients in both the ACLF and CLD-AD groups and $\mathrm{CD} 16^{+}$leukocytes were present at comparable levels in both groups (Figures $5 \mathrm{E}, \mathrm{F} ; \mathrm{p}$ values 0.1508 and 0.1625 respectively). Stratification of ACLF samples based on the presence or absence of sepsis demonstrated higher numbers of $\mathrm{CD} 177^{+}$neutrophils in the ACLF sepsis biopsies although CD $16^{+}$ neutrophils were comparable (Figure $5 \mathrm{G}, \mathrm{H}, \mathrm{p}$ value 0.0635 and $\mathrm{p}$ value 0.2222 respectively).

\section{Pathways Analysis Reveals Several Inflammatory Modules within ACLF}

Pathways analysis using the DEG from ACLF vs CLD and ACLF vs HC were performed using the algorithms ClueGO and Enrichr. ClueGO analysis showed 15 significant pathways for ACLF vs CLD and 12 significant pathways for ACLF vs HC (Supplementary Tables 6 and 7 respectively). Enrichr analysis showed 22 significant pathways for ACLF vs CLD and 18 significant pathways for ACLF vs HC (Supplementary Tables 8 and 9 respectively). Significant enriched pathways included upregulation of inflammatory signatures similar to those observed in well studied inflammatory diseases such as SLE, Asthma, Inflammatory Bowel Disease (IBD) and Rheumatoid Arthritis (RA) (Supplementary Figure 3). GSEA analysis using MSigDB revealed the upregulation of the neutrophil degranulation pathway in ACLF vs CLD, supporting the observation that neutrophil granule genes ELANE and MPO and related cell surface protein CD177 were highly upregulated in ACLF PMN (Supplementary Table 10).

\section{Discussion}

ACLF is driven by a poorly understood innate immune dysfunction which is linked to multiple organ failure and mortality ${ }^{2-10}$. Since neutrophils form a major component of the innate immune system, we investigated transcriptomic signatures of polymorphonuclear cells (PMN) which contain neutrophils, in ACLF patients and compared them with CLD (disease controls) and HC. Our primary findings are discussed below-

1. Overexpression of ELANE, MPO and CD177 in ACLF PMN. We found significantly higher overexpression of ELANE, MPO and CD177 genes in neutrophils from ACLF in comparison to neutrophils in CLD (compensated) and healthy controls (Figure 2F). Over-expression of ELANE, MPO and CD177 in ACLF neutrophils was associated with higher 28-day mortality (Figure 3 A-C). Among the upregulated genes in ACLF , CD177 expression was highest (Figure 2D). Such high CD177 gene upregulation was associated with significant increase in the corresponding neutrophil cell surface CD177 protein expression in ACLF compared to CLD and HC ( $80 \%$ neutrophils in ACLF vs $~ 50 \%$ in CLD vs $\sim 55 \%$ in healthy control; One way ANOVA $p=0.0002$ and ACLF vs CLD $p$ value $<0.0001$ ) as captured by flow cytometric evaluation (Figure 5). The expression of ELANE, MPO and CD177 was not influenced by sex or the presence of bacterial infection (Supplementary Figures 1 and 2). ELANE, MPO and CD177 are among the highest expressed genes in a variety of inflammatory conditions ${ }^{22-}$ 31 . Their high overexpression in ACLF patients as compared to controls, and their association with ACLF non-survivors suggests the involvement of neutrophil responses in ACLF pathogenesis leading 
to mortality. An independent study on ACLF blood immune signatures carried out in a European (French) population has also reported expansion of the $\mathrm{CD}_{177^{+}}$sub-population in $\mathrm{ACLF}^{10}$. This corroborates our observation in a genetically non-identical cohort of ACLF patients. Our study additionally shows that CD177 gene expression is associated with 28-day non-survivors. CD $177^{+}$ neutrophils have been shown to be associated with increased ELANE and MPO gene expression, in agreement with our observations ${ }^{32}$. Therefore ELANE, MPO and CD177 may form a three-gene signature that characterize a pathogenic neutrophil subset in neutrophil mediated pathogenesis in ACLF. Quantitative gene expression values of ELANE, MPO and CD177; or quantitative flowcytometric expression of CD177 on neutrophils may provide quantitative measures for evaluating the extent of inflammation and as a prognostic biomarker for short term mortality.

2. $C D 177^{+}$Neutrophils are present in the liver tissue of deceased ACLF and CLD-AD patients. CD177 is a GPI-anchored cell surface glycoprotein which has been shown to bind the cell adhesion molecule PECAM-1 on endothelial cells ${ }^{33}$. CD177 participates in cell adhesion, tissue transmigration and neutrophil degranulation ${ }^{34}$. However, its exact role in tissue transmigration is controversial and it is not clear whether $\mathrm{CD} 177^{+}$neutrophils are capable of reaching target tissue sites, or not ${ }^{35,36}$. We retrieved archived FFPE liver biopsies from deceased ACLF and CLD-AD patients and carried out dual-colour IHC staining for CD16 and CD177 cell surface proteins. In the liver, CD16 is expressed by all cells of myeloid origin such as neutrophils, macrophages, Kupffer cells, and NK cells and CD177 is a neutrophil specific marker. Therefore $\mathrm{CD} 16^{+} \mathrm{CD} 177^{+}$double positives were scored as neutrophils in the liver biopsies (Figure 5). Liver biopsies isolated from both deceased ACLF and CLD-AD patients were found to have $\mathrm{CD} 177^{+}$neutrophils suggesting that these cells are capable of tissue transmigration (Figure 5). We also observed that in ACLF, patients with sepsis had greater number of $\mathrm{CD} 177^{+}$neutrophils in liver tissues as compared to patients without detectable sepsis (sterile inflammation) (Figure 5G; $p=0.0635$ ). Our observation is corroborated by recent studies carried out in periodontitis as a model for inflammation, that show that $\mathrm{CD}_{177^{+}}$neutrophils preferentially travel towards tissue sites with microbial inflammation as compared to tissue sites with sterile inflammation, although the levels of $\mathrm{CD}_{177^{+}}$neutrophils in circulation are elevated in both ${ }^{35}$. The function of the $\mathrm{CD} 177^{+}$neutrophils is not yet known. However, based on its presence in the liver tissue from our study, along with its reported ability to preferentially get recruited to septic tissues, adhesion to endothelial PECAM1 and, association with increased granule enzyme genes ELANE and MPO, we hypothesize that $C D 177^{+}$neutrophils expressing high levels of cytotoxic enzymes ELANE and MPO reach the liver tissue in severe ACLF. However, the precise role of $\mathrm{CD} 177^{+}$neutrophils, and association with sepsis and the extensive cellular damage that is seen in ACLF, need further investigation ${ }^{35}$. Our study suggests that ACLF 28-day non-survivors have significantly higher levels of $\mathrm{CD} 177^{+}$neutrophils in circulation, indicating a role of the $\mathrm{CD} 177^{+}$neutrophil sub-population in ACLF pathogenesis, that needs to be further explored.

3. Upregulation of Inflammatory Pathways in ACLF PMN. Pathways analysis of overall transcriptomic signatures in ACLF vs CLD revealed the upregulation of inflammatory modules in ACLF PMN that 
resembled gene expression modules in several inflammatory diseases such as SLE, RA and IBD suggesting commonalities in PMN activation in these diseases (Supplementary Figure 3). Neutrophil degranulation pathway was a part of the shared gene signature among the inflammatory diseases and may provide a basis for a common therapeutic target in inflammatory diseases that share this signature (Supplementary Table 10).

A limitation of our study is the limited sample sizes included in various experiments. However, the observations made in the study, particularly in the context of ELANE, MPO and CD177, are supported by significant trends in spite of low sample sizes and therefore, cannot be ignored. We report novel associations between neutrophil-specific genes and ACLF outcomes. We also address the controversial question about the ability of $\mathrm{CD} 177^{+}$neutrophils to transmigrate to sites of tissue injury. Our study shows that they can indeed travel to the liver tissue in deceased ACLF and CLD-AD patients.

A major challenge in ACLF research is the heterogeneity of the disease in terms of variable clinical definitions of ACLF across continents, chronic and acute etiologies, presence or absence of sepsis and the involvement of multi-organ dysfunction. This complicates studies on the pathogenesis of ACLF due the involvement of different kinds of PAMPS and DAMPS, interaction of different molecular and physiological processes ${ }^{37}$. Therefore, it is difficult to extrapolate findings from one study to another and this has been a major challenge in ACLF biomarker research ${ }^{38,39}$. However, our observations which are made in an Indian cohort are corroborated by published studies carried out on genetically non-identical French population, particularly in the context of overexpression of neutrophil CD $177^{10}$. This suggests that neutrophil specific CD177 expression may be a major conserved pathogenic signature in ACLF. While further investigations are needed to understand the mechanistic relevance of these genes and pathways in ACLF; our study provides a rational basis for further exploration of the ELANE, MPO, CD177 signature as potential biomarkers in predicting ACLF outcomes; as well as highlights these neutrophil granule genes and pathways as possible points of therapeutic intervention in ACLF.

\section{Declarations}

\section{Author Contributions:}

PA: designed and supervised study, analyzed and interpreted data, drafted manuscript, acquired funding; RS: acquired, analyzed and interpreted data, drafted manuscript; S.: recruited and managed patients, collected samples, carried out clinical data analysis, provided critical inputs into manuscript; PD, SSP, PM, PD, VS: acquired, analyzed and interpreted data and drafted critical portions of the manuscript. The study sponsors had no role in the study design, implementation or data analysis. All the authors declare no conflict of interest. All authors have approved the final submitted version of the manuscript.

\section{Funding}

This work has been funded by the All India Institute of Medical Sciences New Delhi Intramural Grant 2016 [A-454] and Department of Biotechnology, Government of India [BT/PR18574/BIC/101/151]. RSa was 
supported by fellowship from Indian Council of Medical Research [Fellowship no.JRF-

2016/HRD/LS/98/40800]. The study sponsors had no role in the study design, implementation, collection of data, data analysis or interpretation.

\section{References}

1. Sarin, S. K. et al. Acute-on-chronic liver failure: consensus recommendations of the Asian Pacific Association for the study of the liver (APASL). Hepatology international. 3 (1), 269-282 (2009).

2. Moreau, N. et al. Neutrophil-to-lymphocyte ratio predicts death in acute-on-chronic liver failure patients admitted to the intensive care unit: a retrospective cohort study. Shock (Augusta, Ga.). 49 (4), 385-392 (2018).

3. Fan, Z. et al. Neutrophil-lymphocyte ratio predicts short term mortality in patients with hepatitis B virus-related acute-on-chronic liver failure treated with an artificial liver support system. PloS one. 12 (4), (2017). e0175332

4. Rice, J. et al. Neutrophil-to-Lymphocyte ratio associates independently with mortality in hospitalized patients with cirrhosis. Clinical Gastroenterology and Hepatology. 16 (11), 1786-1791 (2018).

5. Forrest, E. H. et al. Baseline neutrophil-to-lymphocyte ratio predicts response to corticosteroids and is associated with infection and renal dysfunction in alcoholic hepatitis. Alimentary pharmacology \& therapeutics. 50 (4), 442-453 (2019).

6. Bernsmeier, C. et al. Leucocyte ratios are biomarkers of mortality in patients with acute decompensation of cirrhosis and acute-on-chronic liver failure. Aliment. Pharmacol. Ther. 52 (5), 855-865 (2020).

7. Makkar, K. et al. Neutrophil dysfunction predicts 90 day survival in patients with acute on chronic liver failure: A longitudinal case-control study. JGH. 4, 4595-4602 (2020).

8. Khanam, A. et al. Blockade of neutrophil's chemokine receptors CXCR1/2 abrogate liver damage in acute-on-chronic liver failure. Frontiers in immunology. 8, 464 (2017).

9. Wu, W., Sun, S. \& Wang, Y. et. al. Circulating Neutrophil Dysfunction in HBV-Related Acute-on-Chronic Liver Failure. Frontiers in Immunology. 12, 620365 (2021).

10. Weiss, E., de la Grange, P. \& Defaye, M. et.al.,R Characterization of Blood Immune Cells in Patients With Decompensated Cirrhosis Including ACLF. Frontiers in Immunology. 11, 619039 (2021).

11. Gustot, T. \& Moreau, R. Acute-on-chronic liver failure vs. traditional acute decompensation of cirrhosis. J Hepatol. 69 (6), 1384-1393 (2018 Dec).

12. Saito, Y. et al. The influence of blood glucose on neutrophil function in individuals without diabetes. Luminescence. 28 (4), 569-573 (2013).

13. Alba-Loureiro, T. C., Hirabara, S. M., Mendonça, J. R., Curi, R. \& Pithon-Curi, T. C. Diabetes causes marked changes in function and metabolism of rat neutrophils. J Endocrinol. 188 (2), 295-303 (2006). 
14. Stegenga, M. E. et al. Hyperglycemia enhances coagulation and reduces neutrophil degranulation, whereas hyperinsulinemia inhibits fibrinolysis during human endotoxemia. Blood. 112 (1), 82-89 (2008).

15. Sarin, S., Kumar \& Ashok Choudhury. Acute-on-chronic liver failure. Current gastroenterology reports. 18 (12), 1-16 (2016).

16. Boyum, A. Separation of leucocytes from blood and bone marrow. Scand. J. Clin. Invest. 21 (Suppl 97), 77-83 (1968).

17. Xia, J., Gill, E. E. \& Hancock, R. E. W. NetworkAnalyst for statistical, visual and network-based metaanalysis of gene expression data. Nat Protoc. 10, 823-844 https://doi.org/10.1038/nprot.2015.052 (2015).

18. Bindea, G. et al. ClueGO: a Cytoscape plug-in to decipher functionally grouped gene ontology and pathway annotation networks. Bioinformatics. 25, 1091-1093 (2009).

19. Chen, E. Y. et al. Ma'ayan A. Enrichr: interactive and collaborative HTML5 gene list enrichment analysis tool. BMC Bioinformatics. 14, 128 (2013).

20. Xie, Z. et al. Gene Set Knowledge Discovery with Enrichr.Current Protocols(2021)1:e90.

21. Horwitz, M. S., Laurino, M. Y. \& Keel, S. B. Normal peripheral blood neutrophil numbers accompanying ELANE whole gene deletion mutation. Blood Advances. 3 (16), 2470-2473 (2019).

22. Korkmaz, B., Horwitz, M. S., Jenne, D. E. \& Gauthier, F. Neutrophil elastase, proteinase 3, and cathepsin G as therapeutic targets in human diseases. Pharmacol. Rev. 62 (4), 726-759 (2010).

23. Klebanoff, S. J. Myeloperoxidase: friend and foe. Journal of leukocyte biology. 77 (5), 598-625 (2005).

24. Stroncek, D. Neutrophil-Specific Antigen HNA-2a (NB1, CD177): Serology, Biochemistry, and Molecular Biology. Vox sanguinis. 83, 359-361 (2002).

25. Ahmad, S. et al. Transcriptome meta-analysis deciphers a dysregulation in immune responseassociated gene signatures during sepsis. Genes. 10 (12), 1005 (2019).

26. Aschenbrenner, A. C. et al. Disease severity-specific neutrophil signatures in blood transcriptomes stratify COVID-19 patients. Genome Med. 13 (1), 1-25 (2021).

27. Chowdhury, C. S. et al. Enhanced neutrophil extracellular trap generation in rheumatoid arthritis: analysis of underlying signal transduction pathways and potential diagnostic utility. Arthritis research \& therapy. 16 (3), 1-4 (2014).

28. Wang, W., Jian, Z., Guo, J. \& Ning, X. Increased levels of serum myeloperoxidase in patients with active rheumatoid arthritis. Life sciences. 117 (1), 19-23 (2014).

29. Hu, N. et al. Coexpression of CD177 and membrane proteinase 3 on neutrophils in antineutrophil cytoplasmic autoantibody-associated systemic vasculitis: Anti-proteinase 3-mediated neutrophil activation is independent of the role of CD177-expressing neutrophils. Arthr. Rhuem. 60 (5), 15481557 (2009). 
30. Chowdhury, C. S. et al. Enhanced neutrophil extracellular trap generation in rheumatoid arthritis: analysis of underlying signal transduction pathways and potential diagnostic utility. Arthritis research \& therapy. 16 (3), 1-4 (2014).

31. Weiss, E. et al. Characterization of Blood Immune Cells in Patients with decompensated cirrhosis including ACLF. Frontiers in immunology. 11, 3702 (2020).

32. Hu, N. et al. Differential expression of granulopoiesis related genes in neutrophil subsets distinguished by membrane expression of CD177. PLoS One. 9 (6), e99671 (2014).

33. Jerke, U. et al. Complement receptor Mac-1 is an adaptor for NB1 (CD177)-mediated PR3-ANCA neutrophil activation. Journal of biological chemistry. 286, 97070-97081 (2011).

34. Bai, M. et al. CD177 modulates human neutrophil migration through activation-mediated integrin and chemoreceptor regulation. Blood. 130, 2092-2100 (2017).

35. Dahlstrand Rudin, A. et al. The neutrophil subset defined by CD177 expression is preferentially recruited to gingival crevicular fluid in periodontitis. Journal of Leukocyte Biology. 109 (2), 349-362 (2021).

36. Rudin, A. D. et al. The neutrophil subset defined by CD177 expression is preferentially recruited to gingival crevicular fluid in periodontitis. J Leukoc Biol. 109, 349-362 (2021).

37. Martin-Mateos, R., Alvarez-Mon, M. \& Albillos, A. Dysfunctional immune response in acute-on-chronic liver failure: it takes two to tango. Frontiers in immunology. 10, 973 (2019).

38. Kim, T., Yeob \& Kim, D. J. Acute-on-chronic liver failure. Clinical and molecular hepatology. 19, 43494359 (2013).

39. Amin, A. \& Mookerjee, R. P. Acute-on-chronic liver failure: definition, prognosis and management. Frontline Gastroenterol. 11 (6), 458-467 (2019).

\section{Table}

Table 1. Baseline characteristics of ACLF, CLD and HC incorporated in the microarray analysis. 


\begin{tabular}{|c|c|c|c|}
\hline Variables & ACLF $(n=10)$ & CLD $(n=6)$ & $\begin{array}{c}\mathrm{HC} \\
(\mathrm{n}=4)\end{array}$ \\
\hline \multirow{2}{*}{$\begin{array}{c}\text { Age/ years } \\
\text { (Mean } \pm \text { S.D.) } \\
\text { Gender (male) }\end{array}$} & $44( \pm 16)$ & $39( \pm 16)$ & $\begin{array}{l}44( \pm \\
9)\end{array}$ \\
\hline & 10 & 6 & 2 \\
\hline $\begin{array}{l}\text { Etiology } \\
\text { chronic }\end{array}$ & Alcohol (4), AIH (3), Cryptogenic (3), Viral (0) & $\begin{array}{l}\text { Viral (3), } \\
\text { Alcoholic (3) }\end{array}$ & - \\
\hline Etiology acute & $\begin{array}{c}\text { Alcohol (2), Sepsis (3), AIH (1), AIH + sepsis (1), } \\
\text { HEV + alcohol (1), Unknown causes (2) }\end{array}$ & - & - \\
\hline
\end{tabular}

Footnotes: ACLF- Acute-on-Chronic Liver Failure; CLD- Chronic liver disease (compensated); HC- Healthy Controls. AIH- Autoimmune Hepatitis; HEV- Hepatitis E Virus

\section{Figures}




\section{A}

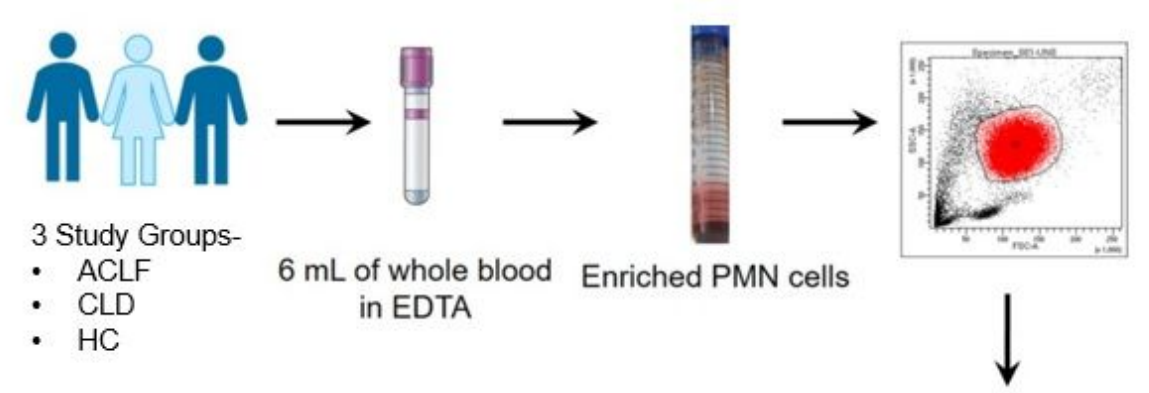

CD16 surface labeling and flow cytometry
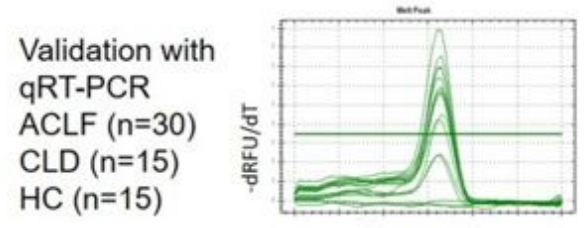

Temperature

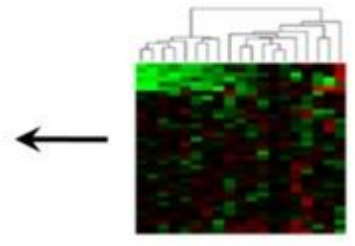

ACLF vs. CLD

ACLF Survivors vs. Non Survivors

ACLF Sepsis vs. Sterile Inflammation

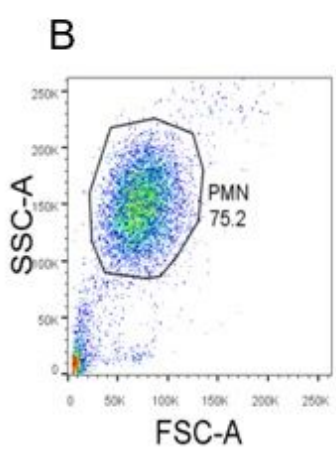

FSC-A

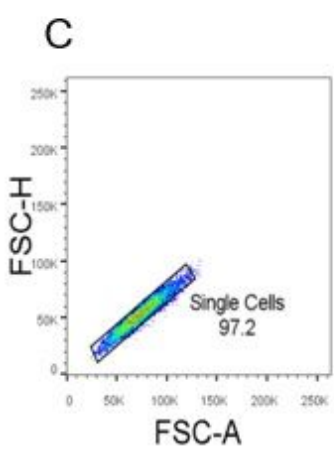

FSC-A

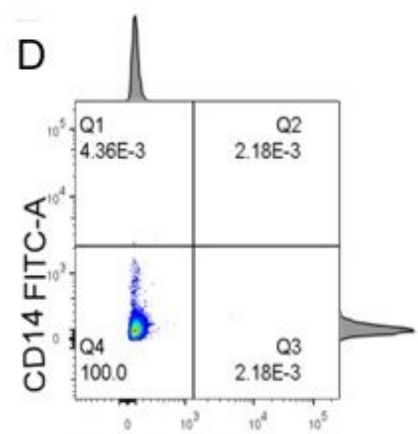

CD16 APC-A
Microarray and Differential Gene Expression Analysis

Samples RIN>6.0; PMN Enrichment $>50 \%$ : $\operatorname{ACLF}(n=10)$ $\operatorname{CLD}(n=6)$ $\mathrm{HC}(\mathrm{n}=4)$

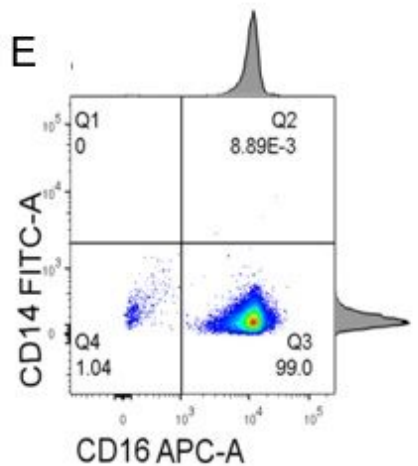

\section{Figure 1}

Overall study design and neutrophil enrichment. (A) Polymorphonuclear neutrophils (PMN) were enriched from the peripheral whole blood of 23 study participants. The isolated PMN were assessed for neutrophil enrichment by CD16 surface staining and analyzed by flow cytometry. RNA were extracted from enriched neutrophils and subjected to microarray analysis followed by quantitative RT-PCR validation of top differentially expressed genes. (B-E) Gating strategy for neutrophil enrichment analysis. (B)The top enriched population (PMN) in FSC vs. SSC dot plot was gated, (C)Single cells were selected in FSC-H vs FSC-A dot plot (D)Unstained cells were gated in CD14-FITC vs. CD16-APC (E) Gating based on unstained cells was used to select CD16+ cells in surface labelled samples. 
A

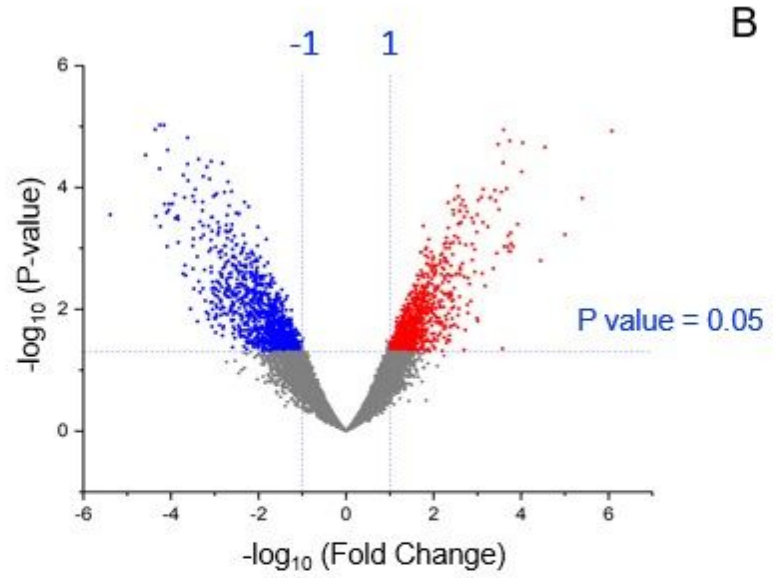

$\mathrm{C}$

\begin{tabular}{|c|c|c|c|c|c|c|c|}
\hline \multicolumn{4}{|c|}{ ACLF vs CLD } & \multicolumn{4}{|c|}{ ACLF vs HC } \\
\hline Symbols & $\log \mathrm{FC}$ & Symbols & $\log F C$ & Symbols & $\log F \mathrm{C}$ & Symbols & $\log \mathrm{FC}$ \\
\hline TRIM58 & -4.5784 & TAAR1 & 2.3348 & 8 FCER1A & -6.0881 & FCER1G & 2.792 \\
\hline CAVIN2 & -4.3606 & ECHDC3 & 2.4777 & 7 CAVIN2 & -5.6224 & NLRC4 & 3.0399 \\
\hline TUBB1 & -4.2585 & ETS2 & 2.5568 & 3 TUBB1 & -5.1816 & 5 SMARCD3 & 3.1296 \\
\hline PRKAR2B & -4.2526 & IRAK3 & 2.5721 & I WDR86-AS1 & -4.8982 & ST6GALNAC3 & 3.1583 \\
\hline MYBL1 & -4.1576 & ALDH2 & 2.6281 & 1 NELLZ2 & -4.7433 & 3 ECHDC3 & 3.2582 \\
\hline CXCR2P1 & -4.0754 & SMARCD3 & 2.6682 & 2 FAM 153 CP & -4.731 & PFKFB2 & 3.3516 \\
\hline PF4V1 & -3.9104 & $\mathrm{VCX} 2$ & 2.7271 & I PRKAR2B & -4.7157 & 7 GADD45A & 3.3733 \\
\hline CD2AP & -3.7169 & SMPDLIA & 3.0272 & 2 LINC00892 & -4.5941 & L GYG1 & 3.3846 \\
\hline C20rf88 & -3.6165 & LHX1 & 3.0983 & $3 \mathrm{KIF5C}$ & -4.5776 & PIASZ & 3.4551 \\
\hline ALDH5A1 & -3.615 & HPN & 3.1369 & 9 MYBL1 & -4.575 & ; IRAK3 & 3.6201 \\
\hline TENTSC & -3.6078 & IER3 & 3.2899 & CXCR2P 1 & -4.5435 & ; FKBPS & 3.6218 \\
\hline GNG11 & -3.5007 & ELANE & 3.3036 & 5 RBPMSZ & -4.4868 & 3 CLEC4D & 3.6879 \\
\hline RNF144A & -3.4424 & CCL3L3 & 3.4072 & 2 MAP $3 K 7 C L$ & -4.4783 & VNN1 & 3.9743 \\
\hline HERC6 & -3.368 & TDRD9 & 3.4226 & 5 KLRG1 & -4.3494 & 4 LHXI & 4.0766 \\
\hline NT5M & -3.2616 & GRB10 & 3.4782 & 2 FAM153B & -4.3269 & TPST 1 & 4.2301 \\
\hline TAL1 & -3.1788 & PRL & 3.5556 & 5 RORC & -4.3059 & MCEMP 1 & 4.3386 \\
\hline PBX1 & -3.1152 & SLCO $4 A 1$ & 3.5924 & 4 ZNF204P & -4.2505 & DAAMZ & 4.353 \\
\hline PNPT1 & -3.0773 & VNN1 & 3.6082 & 2 GNAZ & -4.2408 & 3 SLCO $4 A 1$ & 4.5811 \\
\hline GNAZ & -3.0263 & ILIR2 & 3.6694 & 4 TCF7 & -4.21 & L TDRD9 & 4.5983 \\
\hline AGRN & -2.9974 & DAAMZ & 3.7418 & 8 ELOVL7 & -4.0388 & 3 SLC51A & 4.7019 \\
\hline JAMB & -2.9554 & COL17A1 & 4.0159 & NR3C2 & -3.8211 & GRB10 & 4.7979 \\
\hline TRMT11 & -2.8214 & OLFM4 $\leftarrow$ & 4.0405 & 5 IGFBP3 & -3.7834 & + C11orf96 & 4.8932 \\
\hline CALD1 & -2.7758 & C11orf96 & 4.5485 & $5 \mathrm{MMD}$ & -3.6935 & ARG1 & 6.321 \\
\hline GFPT 2 & -2.6912 & $\mathrm{CD} 177 \leftarrow$ & 5.3977 & 7 PI 16 & -3.6368 & 3 CD177 $\leftarrow$ & 7.4834 \\
\hline$\pi C 7 B$ & -2.6159 & $\mathrm{OLAH} \leftarrow$ & 6.0812 & RCAN3 & -3.2266 & 5 OLAH & 7.745 \\
\hline
\end{tabular}
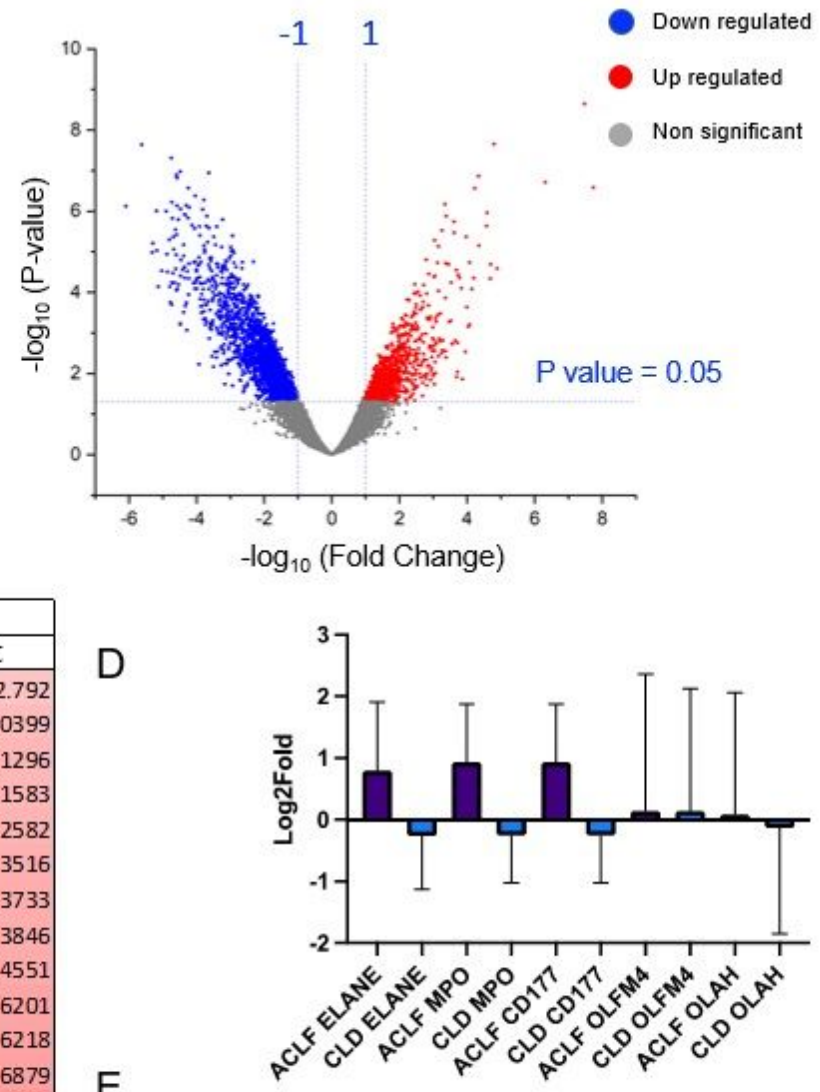

E

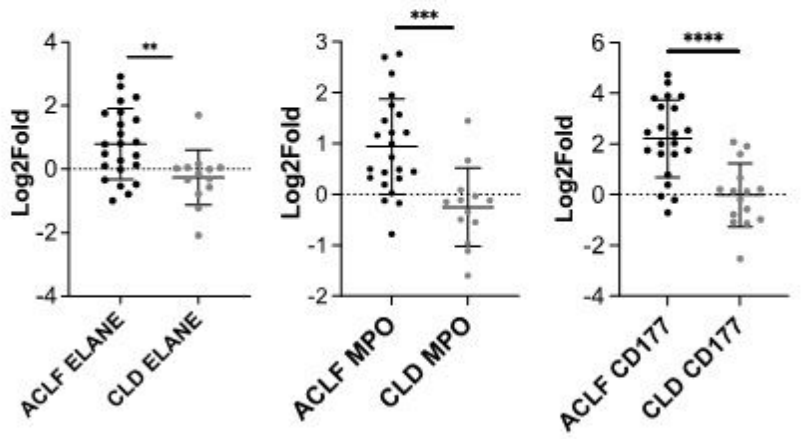

\section{Figure 2}

Total gene expression analysis and differentially expressed genes. (A) Volcano plots representing -log ( $p$ value) vs log fold change of ACLF vs HC, ACLF vs CLD. (B) Two-way analysis for differential gene expression. Top 50 upregulated and downregulated genes represented as heatmap with respective log fold changes (B) ACLF vs HC and (C) ACLF vs CLD. (D) Validation of microarray gene expression trends by qRT-PCR. Y-axis represents Mean log fold change of differentially expressed genes in ACLF vs. CLD and $\mathrm{X}$-axis represents gene names. Validation with qRT-PCR was performed for the following genes: ELANE, MPO, CD177, OLFM4, OLAH. Sample sizes were ACLF $(n=10), \operatorname{CLD}(n=3), H C(n=4)$ for microarray and ACLF ( $n=30)$, CLD $(n=15)$ and HC $(n=15)$ for qRT-PCR. (E) Pairwise analysis shows significant elevation of ELANE ( $p$ value $=0.0064)$, MPO ( $p$ value $=0.0003$ ) and CD177 ( $p$ value $<0.0001)$ in ACLF vs CLD. 

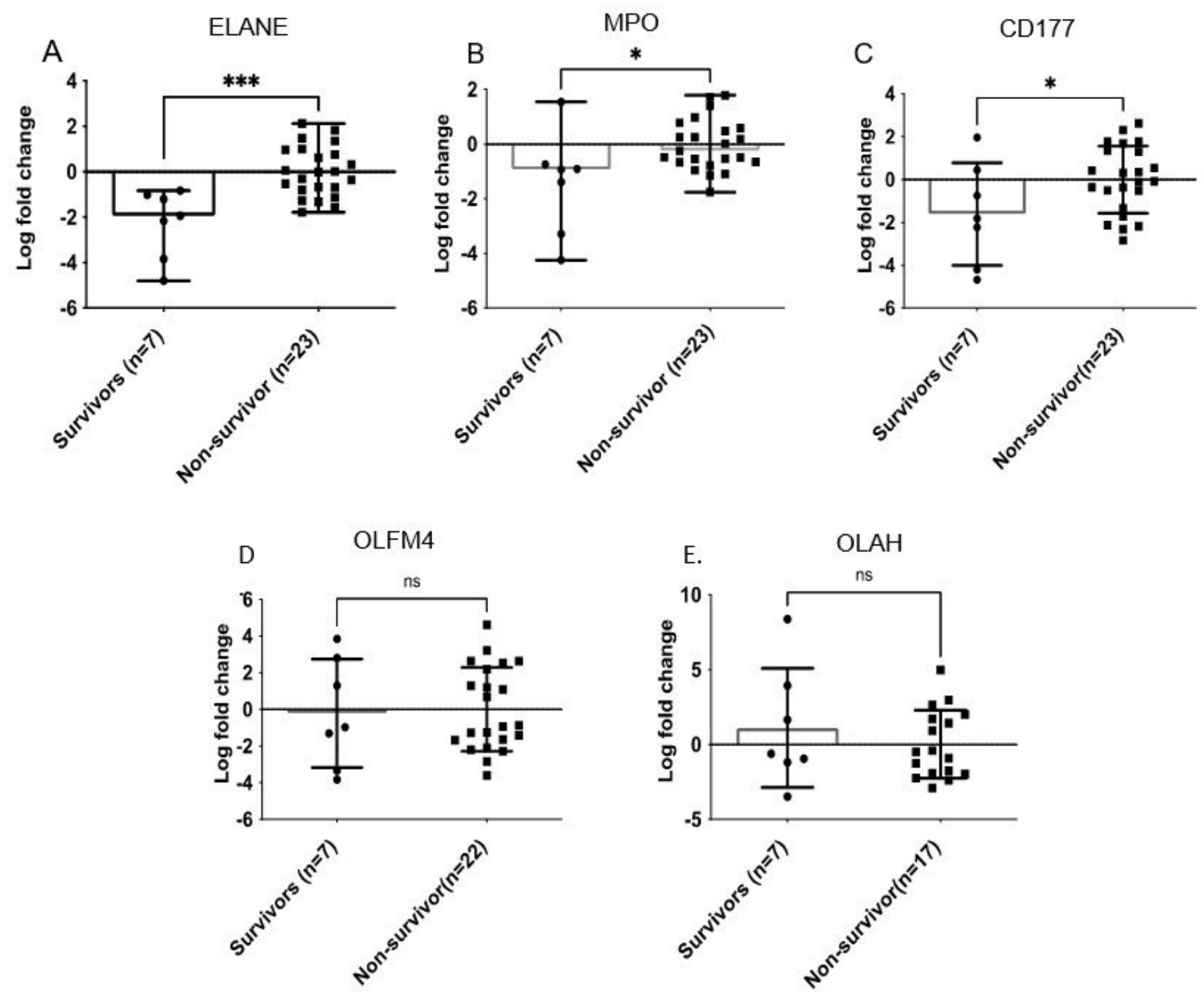

Figure 3

Differential gene expression of ELANE, MPO and CD177 in ACLF 28-day non-survivors vs. survivors. Gene expression assessment of selected differentially expressed genes by qRT-PCR in ACLF 28-day nonsurvivors vs. survivors. For the calculation of relative expression and log fold change, 18 s rRNA was taken as reference gene and survivors were taken as calibrator group. ELANE, MPO and CD177 were found to be elevated in 28-day non-survivors. Unpaired t-test was used for statistical testing; (A) ELANE ( $p$ value $=0.004),(B)$ MPO ( $p$ value $=0.01),(C)$ CD177 ( $p$ value $=0.03),(D)$ OLFM4 ( $p$ value= 0.12), (E) OLAH ( $p$ value $=0.67) .0 .004,0.02,0.04$ 

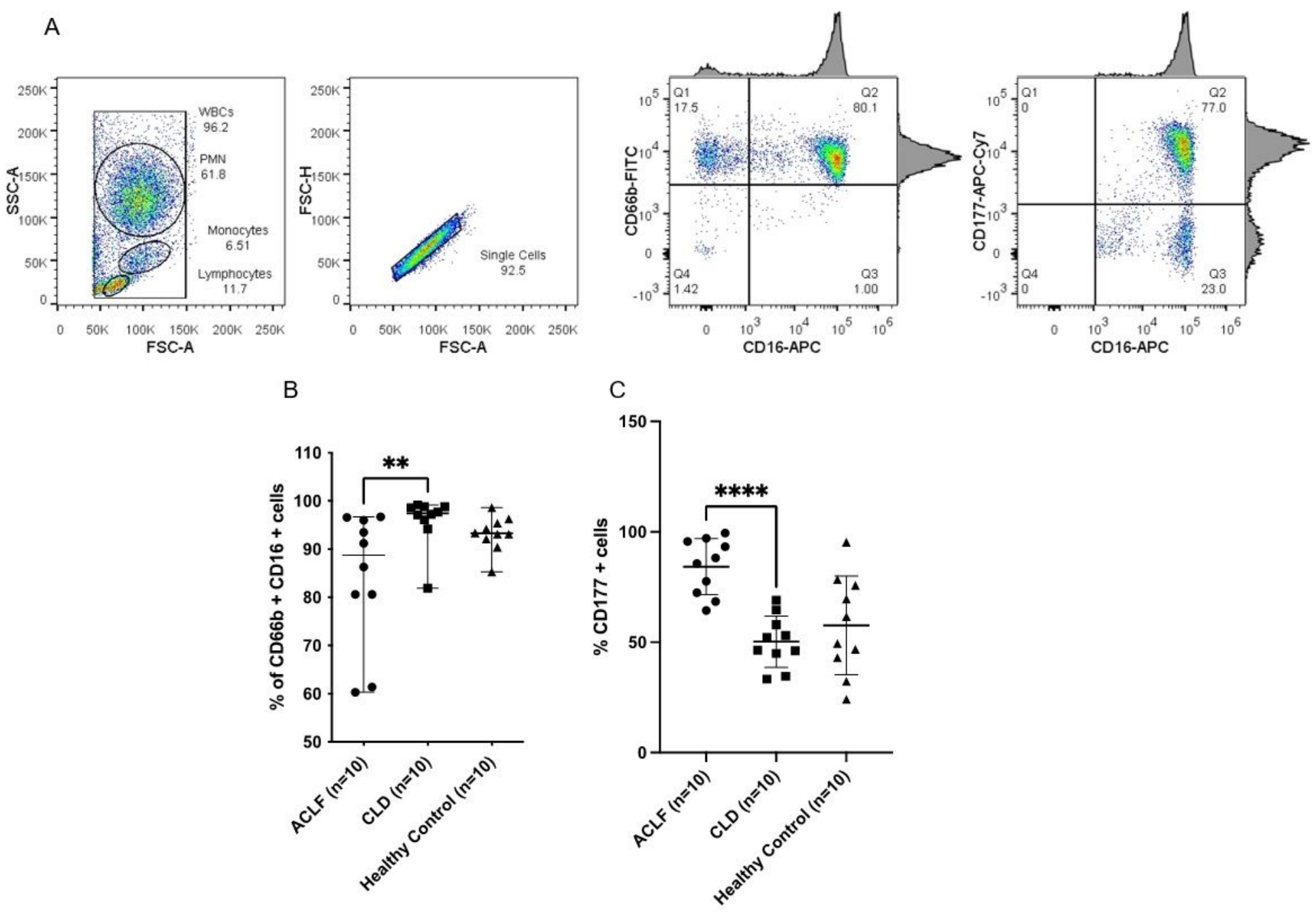

\section{Figure 4}

CD177 cell surface level expression is higher in ACLF vs CLD. (A) Gating strategy for assessment of CD17+ cells by flow cytometry. Neutrophil enrichment was determined by CD66b+ and CD16+positivity. The first plot represents an FSC vs SSC scatter plot, used for gating PMN cells. Singlets gating as determined by FSC-height v/s FSC-area plot. This is followed by a quadrant plot for CD66b-FITC vs CD16APC, and double positive neutrophils are gated on Q2. The last quadrant plot represents CD177-APC-Cy7 vs CD16-APC. CD177 positive cells are gated. (B) CD66b+CD16+ positive neutrophil percentage across groups. The mean percentage of enrichment is significantly different across groups as determined by Kruskal Wallis test (C) CD177 positive cell percentage across study groups. The mean CD177 population was found to be significantly different across the three groups, as calculated by one-way ANOVA. Pairwise analysis for CD177+ cell percentages were performed for ACLF vs CLD. *p- value $\leq 0.05$; ${ }^{*} \mathrm{p}$ value $\leq 0.005,{ }^{* \star *} \mathrm{p}$ - value $\leq 0.001$ 

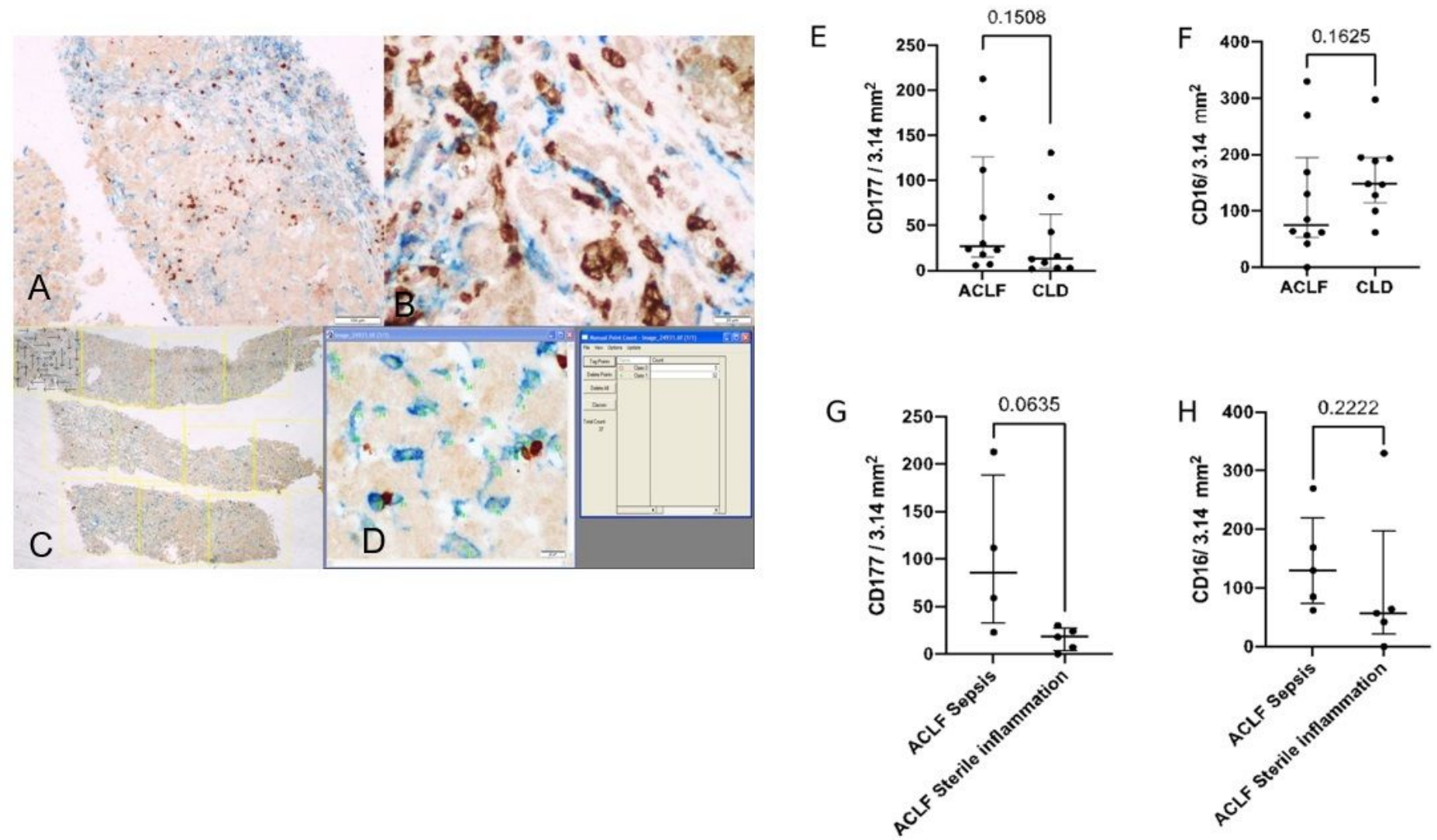

\section{Figure 5}

Immunohistochemistry of post-mortem biopsies obtained from ACLF and CLD-AD patients. (A-B) The dual-colour stained slides were photographed by using a BX43 Olympus microscope. The CD177 stained neutrophils were represented by brown stain (black arrows) and the CD16 stained neutrophils, monocytes, macrophages, and Kupffer cells were represented by blue stain (blue arrows) (A x40, B x 400). (C) Method for observation of dual-colour stained slide under $2 x$ objective of the microscope and selection of nonoverlapping FOVs followed by counting at $\times 40$; (D) Method of using the manual tagging and counting tool of the Image Proplus 6.1 software [D x40] (E) CD177+ Neutrophil count/ 3.14 mm2 in ACLF and CLDAD patients; (F) CD16+ leukocytes in ACLF and CLD-AD patients; (G) CD177+ Neutrophil count/ 3.14 $\mathrm{mm} 2$ in ACLF patients with or without sepsis; $(H)$ CD16+ leukocytes in ACLF and CLD-AD patients. Sample size for each group- ACLF ( $n=10)$; CLD-AD ( $n=9)$; ACLF sepsis $(n=4)$; ACLF sterile inflammation $(n=5)$. For 1 ACLF sample, categorization as sepsis/ sterile inflammation was not available). Nonparametric Mann Whitney Test was performed to determine $p$ values (indicated on the top of the graphs). Graphs are plotted as Median with IQR.

\section{Supplementary Files}

This is a list of supplementary files associated with this preprint. Click to download. 
- SupplementaryFigure1.pptx

- SupplementaryFigure2.pptx

- SupplementaryFigure3pathways.pptx

- SupplementaryTable1apt.docx

- SupplementaryTable2DGEACLFvsCLD.xlsx

- SupplementaryTable3DGEACLFvsHC.xlsx

- SupplementaryTable4CD177quantitation.docx

- SupplementaryTable5CD177IHCtable.xlsx

- SupplementaryTable6ClueGOResultTableACLFvsCLD.xls

- SupplementaryTable7ClueGOResultTableACLFvsHC.xls

- SupplementaryTable8ACLFvsCLDEnrichrKEGG.xls

- SupplementaryTable9ACLFvsHCEnrichrKEGG.xls

- SupplementaryTable10MSIGDBGSEAACLFvsCLDoverlapcopy.numbers 